\title{
Informações sobre hipertensão arterial emergentes de usuários cadastrados no programa HiperDia
}

\author{
Aldaíza Ferreira Antunes Fortes, M.Sc.*, Ana Maria Nassar Cintra Soane, M.Sc.**, \\ Karla Karollyne Ferreira Vieira Ramos***, Poliana Aparecida Gomes Ferreira***
}

${ }^{*}$ Enfermeira, Docente do Estágio Supervisionado e Responsável pelo Setor de Pesquisa da EEWB, ${ }^{* *}$ Coordenadora do Departamento de Ensino e Pesquisa da EEWB, ***Enfermeiras, EEWB - Extraído do Trabalho de Conclusão de Curso de Graduação em Enfermagem da Escola de Enfermagem Wenceslau Braz (EEWB) intitulado "Perfil epidemiológico e informaçôes sobre hipertensão arterial emergentes de usuários cadastrados no programa HiperDia"

\begin{abstract}
Resumo
Trata-se de um estudo de abordagem qualitativa, do tipo transversal, descritivo e exploratório. Os objetivos foram conhecer os significados de hipertensão arterial para os hipertensos cadastrados no programa HiperDia da cidade de Itajubá/MG, os danos causados à saúde e avaliar se esses pacientes seguem o tratamento para o qual são orientados. A pesquisa foi realizada com 25 sujeitos, 17 do gênero feminino e 8 masculino, em cinco Unidades Básicas de Saúde do município. Os dados foram descritos sob referencial das Representaçôes Sociais, utilizando o Discurso do Sujeito Coletivo. Prevaleceram as seguintes ideias centrais "Nervosismo e preocupaçâo", "Alteração vascular" e "Hábitos irregulares" na primeira questão, "Responsável por doenças secundárias" e "Transtornos nas atividades da vida diária” na pergunta seguinte e "Manter a saúde para viver" na última questão. É preciso que o enfermeiro conheça os seus clientes e adapte as orientaçóes de enfermagem para cada realidade vivenciada.
\end{abstract}

Palavras-chave: hipertensão arterial, representaçôes sociais, discurso do sujeito coletivo.

\section{Abstract \\ Information on arterial hypertension in registered users of HiperDia program}

This is a qualitative, cross-sectional descriptive and exploratory study. The objectives were to know the meanings of hypertension for patients enrolled in the HiperDia program in Itajubá/MG, the damage caused to health, and to evaluate if these patients are following the treatment. The survey was conducted with 25 subjects, 17 female and 8 male, in five Basic Health Units of the city. Data were described in Social Representations reference, using the Collective Subject Discourse. The following central ideas prevailed: "Nervousness and worry", "Vascular alteration" and "Irregular habits" in the first issue; "Responsible for secondary diseases" and "Disorders in activities of daily life" in the following question; and "Maintaining a healthy lifestyle" in the last question. It is needed that nurses know their clients and adapt the guidelines for each experienced reality.

Key-words: hypertension, social representations, collective subject discourse. 


\section{Resumen}

\section{Informaciones sobre hipertensión arterial de usuarios registrados en el programa HiperDia}

Se trata de un estudio cualitativo, transversal, descriptivo y exploratorio. Los objetivos fueron conocer los significados de la hipertensión para los hipertensos del programa HiperDia de la ciudad de Itajubá/MG, daños causados a la salud y evaluar si estos pacientes siguen el tratamiento prescrito. La investigación se realizó con 25 sujetos, 17 del género femenino y ocho del masculino, en cinco Unidades Básicas de Salud de la ciudad. Los datos fueron descritos bajo el punto de referencia de las Representaciones Sociales, con el Discurso del Sujeto Colectivo. Las siguientes ideas principales prevalecieron: "Nerviosismo y preocupación", "Alteraciones vasculares" y "Hábitos irregulares" en la primera cuestión, «Responsables por enfermedades secundarias» $\mathrm{y}$ «Trastornos en las actividades de la vida diaria» en la siguiente y "Mantener salud para vivir» en la última. Es necesario que las enfermeras conozcan a sus clientes y adapten las directrices para cada realidad de enfermería experimentada.

Palabras-clave: representaciones sociales, discurso del sujeto colectivo, hipertensión.

\section{Introdução}

Há algumas décadas as doenças do aparelho circulatório são a principal causa de morte no Brasil, sendo que no ano de 2000 corresponderam a mais de $27 \%$ do total de óbitos. Nesse sentido, a Hipertensão Arterial (HA) e o Diabetes Mellitus (DM) constituem os principais fatores de risco para as doenças cardiovasculares. $\mathrm{O}$ acompanhamento e o controle dessas patologias no âmbito da atenção básica poderão evitar o surgimento e a progressão das complicaçôes, reduzindo o número de internaçóes hospitalares, bem como a mortalidade devido a esses agravos [1].

Nesta perspectiva desenvolveu-se o programa governamental HiperDia. Esse é um sistema de cadastramento e acompanhamento de hipertensos e diabéticos captados no Plano Nacional de Reorganização da Atenção à HA e ao DM, realizado em todas as unidades ambulatoriais do Sistema Único de Saúde (SUS), gerando informaçóes para os gerentes locais, gestores das secretarias municipais, estaduais e Ministério da Saúde [2].

Segundo dados da Sociedade Brasileira de Hipertensão [3], a HA atinge $30 \%$ da população adulta brasileira, chegando a mais de $50 \%$ na terceira idade e está presente em $5 \%$ das crianças e adolescentes no Brasil, sendo responsável por $40 \%$ dos infartos, $80 \%$ dos acidentes vasculares encefálicos e $25 \%$ dos casos de insuficiência renal terminal.

Diante das evidências estatísticas, torna-se imprescindível a adesão do hipertenso ao tratamento farmacológico e não-farmacológico, já que a $\mathrm{HA}$ é uma doença crônica grave e que deixa sequelas irreversíveis nos indivíduos que não mantêm os seus níveis pressóricos em taxas adequadas.

De acordo com Machado, Stipp e Leite [4], devido à gravidade do problema e a ausência de um diagnóstico precoce, a melhor forma de combater a HA é a intervenção primária.

Nesse sentido, o enfermeiro tem participação ativa, com caráter de persuasáo, levando o paciente a modificar seus hábitos e a encarar o tratamento como um fator que propicie a melhora da qualidade de vida.

Assim sendo, além de observarmos que a falta de adesáo ao tratamento continua sendo um dos problemas relacionados à $\mathrm{HA}$, percebemos que a assistência prestada ao paciente hipertenso, em muitos casos, não tem sido ampla, ou seja, o paciente náo está sendo atendido de maneira sistêmica.

Portanto, o estudo teve como objetivos: conhecer os significados de HA e os danos causados à saúde para os pacientes portadores desta doença cadastrados no programa HiperDia do município de Itajubá-MG; avaliar se esses pacientes seguem o tratamento para o qual são orientados.

\section{Material e métodos}

Trata-se de um estudo qualitativo, do tipo transversal, descritivo e exploratório. Utilizou-se como cenário de estudo o município de Itajubál $\mathrm{MG}$, sendo que a pesquisa foi realizada em cinco bairros, em suas respectivas Unidades Básicas de Saúde (UBS), a saber: Novo Horizonte, Santa Rosa, Cruzeiro, Rebourgeon e Sáo Vicente. 
O estudo teve como sujeitos portadores de HA cadastrados no Sistema de Cadastramento e Acompanhamento de Pacientes Hipertensos e Diabéticos - programa HiperDia.

A amostra foi composta por 25 sujeitos com média de idade de 63 anos, sendo 17 do gênero feminino e 8 masculino, selecionados através da amostragem proposital.

Utilizou-se um roteiro de entrevista semiestruturada com questóes que visavam avaliar o conhecimento do portador de HA sobre sua doença, os danos causados a saúde e se realizava o tratamento para o qual é orientado.

Os dados qualitativos foram descritos sob o referencial das Representaçóes Sociais (RS), utilizando o Discurso do Sujeito Coletivo (DSC) como método para construçáo dos significados, permitindo a aproximação com o fenômeno em estudo.

As RS consistem na maneira de interpretar a nossa realidade cotidiana, uma forma de conhecimento social, ao associar a atividade mental desenvolvida pelos indivíduos e os grupos para fixar sua posição em relação à situação, acontecimentos, objetos e comunicaçáo que lhes dizem respeito [5].

Lefrève e Lefrève [6] definem o DSC como uma estratégia metodológica com a finalidade de tornar mais nítida uma determinada RS e o conjunto das representaçóes que constituem um dado imaginário. Por meio desse modo discursivo é RS, na medida em que ela aparece náo sob a forma (artificial) de quadros, tabelas e gráficos, mas sob a forma mais viva e direta de um discurso, que é um modo como os indivíduos reais e concretos pensam.

O presente estudo esteve de acordo com os preceitos estabelecidos pela Resolução número 196/96, do Conselho Nacional de Saúde (CNS), de 10 de outubro de 1996 no que diz respeito às diretrizes e normas regulamentadoras de pesquisas envolvendo seres humanos.

$\mathrm{O}$ anonimato foi preservado atribuindo a cada participante a letra $S$ de sujeito com numeral ordinal representando a sequência da entrevista. Ex.: Sujeito $1-S 1$.

\section{Resultados}

Os dados qualitativos são referentes ao conhecimento do significado da doença pelos portadores de HA, como também aos danos causados à saúde e a adesão ao tratamento por meio das orientaçóes recebidas.

Apresentamos a seguir um quadro-síntese das IC emergidas de cada questáo da entrevista.

O quadro I sinaliza a síntese das IC referentes à $1^{\mathrm{a}}$ questão: "Se alguém lhe perguntasse o que é Pressáo Alta, o que o (a) senhor (a) responderia?"

Quadro I - Ideias centrais referentes ao significado da Hipertensão Arterial.

\begin{tabular}{lcc}
\hline \multicolumn{1}{c}{ Ideias centrais } & Sujeitos & $\begin{array}{c}\text { Frequên- } \\
\text { cia }\end{array}$ \\
\hline $\begin{array}{l}\text { 1. Nervosismo e } \\
\text { preocupação }\end{array}$ & $1,2,3,6,14,21,23$ & 7 \\
$\begin{array}{l}\text { 2. Doença gené- } \\
\text { tica }\end{array}$ & 1,10 & 2 \\
$\begin{array}{l}\text { 3. Conjunto de } \\
\text { sinais e sintomas }\end{array}$ & $3,8,14,16,19$ & 5 \\
$\begin{array}{l}\text { 4. Alteração } \\
\text { vascular }\end{array}$ & $4,7,11,12,13,18$, & 7 \\
$\begin{array}{l}\text { 5. Hábitos irregu- } \\
\text { lares }\end{array}$ & $5,6,10,13,15,17$, & 7 \\
$\begin{array}{l}\text { 6. Doença ruim } \\
\text { pelo risco de do- } \\
\text { ença secundária e } \\
\text { morte }\end{array}$ & $9,13,19,22$ & 4 \\
$\begin{array}{l}\text { 7. Doença crô- } \\
\text { nica }\end{array}$ & $11,14,19,23,25$ & 5 \\
$\begin{array}{l}\text { 8. Uso contínuo } \\
\text { de medicamento } \\
\text { 9. Não sei }\end{array}$ & $12,14,15,20,24$ & 5 \\
\hline TOTAL & 9 & 14 \\
\hline
\end{tabular}

Fonte - Formulário de Pesquisa.

Os Quadros II, III e IV mostram as sínteses das IC referentes à 2a questão: "Para o (a) senhor (a), a Pressão Alta pode afetar a sua saúde? Por quê?"

Quadro II - Ideias centrais referentes ao dano causado à saúde.

\begin{tabular}{lcc}
\hline Ideias Centrais & Sujeitos & $\begin{array}{c}\text { Frequên- } \\
\text { cia }\end{array}$ \\
\hline 1.Sim & $\begin{array}{l}1,2,3,4,5,6,7,8,9,10,1 \\
\\
\end{array}$ & 24 \\
& $9,20,13,14,15,16,18,1$ & \\
2.Não & \multicolumn{2}{c}{$17,23,24,25$} \\
\hline TOTAL & 2 & 1 \\
\hline
\end{tabular}

Fonte - Formulário de Pesquisa. 
Quadro III - Ideias centrais referentes aos sujeitos que afirmaram que a hipertensão arterial pode afetar a saúde.

\begin{tabular}{lcc}
\hline \multicolumn{1}{c}{ Ideias centrais } & Sujeitos & $\begin{array}{c}\text { Frequên- } \\
\text { cia }\end{array}$ \\
\hline $\begin{array}{l}\text { 1.Responsável por } \\
\text { doenças secun- } \\
\text { dárias }\end{array}$ & $1,2,3,6,9,10,11$, & 12 \\
$\begin{array}{l}\text { 2.Uso contínuo de } \\
\text { medicamento }\end{array}$ & $2,20,22,24$ & 4 \\
$\begin{array}{l}\text { 3.Transtornos nas } \\
\text { atividades da vida } \\
\text { diária }\end{array}$ & $\begin{array}{l}18,5,7,20,21,23,25 \\
\text { TOTAL }\end{array}$ & 12 \\
\hline
\end{tabular}

Fonte - Formulário de Pesquisa.

Quadro IV - Ideia central referente ao sujeito que afirmou que a Hipertensão Arterial não pode afetar a saúde.

\begin{tabular}{lcc}
\hline \multicolumn{1}{c}{ Ideias centrais } & Sujeitos & $\begin{array}{c}\text { Frequên- } \\
\text { cia }\end{array}$ \\
\hline $\begin{array}{l}\text { 1.Tipo sanguíneo } \\
\text { bom }\end{array}$ & 17 & 1 \\
\hline TOTAL & 1 & \\
\hline
\end{tabular}

Fonte - Formulário de Pesquisa.

Os Quadros V, VI e VII evidenciam as sínteses das IC referentes à $3^{\text {a }}$ questão: " $O(a)$ senhor (a) realiza o tratamento para o qual é orientado (a)? Por quê??"

Quadro V - Ideias centrais referentes à realização do tratamento.

\begin{tabular}{lcc}
\hline Ideias centrais & Sujeitos & $\begin{array}{c}\text { Frequên- } \\
\text { cia }\end{array}$ \\
\hline 1.Sim & $\begin{array}{l}1,2,3,4,5,7,8,9, \\
\\
\end{array}$ & 21 \\
& $10,11,12,13,14,17$, & \\
& $18,19,20,21,22$, & \\
24,25 & 4 \\
2.Parcialmente & $6,15,16,23$ & \\
\hline TOTAL & \multicolumn{2}{c}{2} \\
\hline
\end{tabular}

Fonte - Formulário de Pesquisa.
Quadro VI - Ideias centrais referentes ao motivo da realização do tratamento.

\begin{tabular}{lcc}
\hline \multicolumn{1}{c}{ Ideias centrais } & Sujeitos & $\begin{array}{c}\text { Frequên- } \\
\text { cia }\end{array}$ \\
\hline $\begin{array}{l}\text { 1.Manter a saúde } \\
\text { para viver }\end{array}$ & $\begin{array}{r}1,2,4,5,9,10,11, \\
12,20,21,24,25\end{array}$ \\
$\begin{array}{l}\text { 2.Em busca de } \\
\text { cura }\end{array}$ & 1 \\
$\begin{array}{l}\text { 3.Não causar } \\
\text { transtorno }\end{array}$ & 8 \\
$\begin{array}{l}\text { 4.Para não } \\
\text { morrer }\end{array}$ & 1 \\
\hline TOTAL & 13 \\
\hline \\
Fonte - Formulário de Pesquisa.
\end{tabular}

Quadro VII - Ideias centrais referentes ao motivo da realização parcial do tratamento.

\begin{tabular}{lcc}
\hline Ideias centrais & Sujeitos & \multicolumn{2}{c}{$\begin{array}{c}\text { Frequên- } \\
\text { cia }\end{array}$} \\
\hline $\begin{array}{l}\text { 1.Não é tão im- } \\
\text { portante }\end{array}$ & 6,23 & 2 \\
$\begin{array}{l}\text { 2.Só o remédio é } \\
\text { suficiente }\end{array}$ & 15 & 1 \\
$\begin{array}{l}\text { 3.Tem que ter } \\
\text { força de vontade }\end{array}$ & 16 & 1 \\
\hline TOTAL & & \\
\hline
\end{tabular}

Fonte - Formulário de Pesquisa.

\section{Discussão}

Os dados qualitativos discutidos a seguir são referentes às IC que emergiram com maior frequência após a realização da pesquisa.

A Hipertensão Arterial Sistêmica, doença cardiovascular de grande interesse para a saúde pública, é largamente conhecida como fator de risco para o desenvolvimento de outras doenças cardiovasculares [7].

Essa visão, no entanto, é frequentemente reduzida às causas que a produzem, rotulando-a como "emocional" e "nervosa". Tal fato poderia ser explicado pela inexistência de uma causa física identificável [8].

Pierin et al. [9] também explanam sobre esse assunto, percebendo um desconhecimento da etiologia da HA e uma concentração de respostas, atribuindo as causas da doença aos aspectos emocionais. Tal fato foi comprovado em nossa pesquisa, referente à $1^{\text {a }}$ questáo, ao questionar os entrevistados sobre a definição de HA, evidenciando-se nos depoimentos a seguir: 
[...] Imagino que a gente fica nervosa com as coisas da vida, a gente fica preocupada né, com as coisas e a pressão sobe (S2).

[...] Pressão alta é de acordo com a irritação da gente, quando a gente fica assim irritado ou tá preocupado com uma coisa e com outra né, por exemplo, se tem uma coisa pra você fazer hoje e não vai dar tempo para você fazer e aquilo é preciso ser feito entáo ali a gente preocupa a cabeça e a pressão vai subindo (S21).

Outra ideia central frequente na primeira questão foi "Alteração vascular". Esse fato é comprovado por Miranda et al. [10] ao discorrerem sobre envelhecimento arterial, afirmando que as alteraçóes das propriedades vasculares da aorta, que ocorrem com o envelhecimento, têm importante papel na gênese $\mathrm{e}$ progressão da hipertensão arterial sistêmica. Um dos depoimentos que surgiu relacionado a esta questáo foi:

Eu sei que pressão alta é coisa que aparece quando a pessoa já tá ficando de idade, porque eu não tinha pressão alta [...] (S12).

Continuando nesta ideia central, observou-se que a fala de um dos participantes, ao definir HA, sobressaiu sobre os demais:

[...] no meu conceito, pressão alta seria, é... Uma pressáo sanguínea que passa dentro da artéria muito maior do que o normal que ela tem que ser [...] (S13).

Esse conceito é comprovado pela definição de HA da Sociedade Brasileira de Hipertensão [3] que classifica como hipertenso o indivíduo acima de 18 anos que apresenta pressão sistólica maior que 140 $\mathrm{mmHg}$ e diastólica maior que $90 \mathrm{mmHg}$, sendo o normal, ao considerar a sistólica abaixo de 130 $\mathrm{mmHg}$ e diastólica $85 \mathrm{mmHg}$.

Ainda na primeira questão, apresentou-se também frequente a ideia central "Hábitos irregulares". Por hábitos irregulares entende-se alimentação inadequada, uso de substâncias como álcool e cigarro e sedentarismo. Esses fatores foram identificados no seguinte discurso:

[...] A pressão alta é a maneira da pessoa se alimentar né, comer muito sal, a bebida né, o álcool, até o cigarro que eu fumo ele pode subir a pressão $(\mathrm{S} 17)$.

Todas essas expressões constituem-se fatores de risco para a HA, segundo a Sociedade Brasileira de Hipertensão [3]. No entanto, a atribuição de hábitos irregulares como conceito de HA confirma o desconhecimento do portador de HA sobre sua doença.

Péres, Magna e Viana [10] deduziram que mudar um estilo de vida, bem como um hábito alimentar pode levar ao isolamento social em função dos pacientes deixarem de ir a festas e reunióes para evitar ingerir alimentos inadequados.

Assim, a condição crônica de saúde pode levar a diversas perdas nos relacionamentos sociais, nas atividades de lazer e de prazer (beber, fumar e comer), nos investimentos financeiros e na capacidade física. Todas essas perdas levam ao desânimo, tristeza e depressão, sendo um agravante no momento de mudar um hábito ou um estilo de vida.

Já em comunidades de baixo nível de renda, não se obedece às orientações, mesmo quando essas são conhecidas. Em relação às mudanças no estilo de vida, para adotarem um mais saudável, essas pessoas têm a justificativa de que o abandono de um dos hábitos prejudiciais pode significar a perda de um prazer, o que é relevante a elas em um contexto de vida no qual as oportunidades de satisfação são escassas [10].

A quarta ideia central frequente a ser discutida em relação à primeira questão refere-se à definição de HA apenas como "Doença crônica". Uma condição crônica de saúde caracteriza-se pelo momento em que a pessoa passa a incorporar a doença no seu processo de viver, constituindo-se em situação permeada de estresse. Seu impacto surge a qualquer tempo e para permanecer, com capacidade de causar alteraçóes nas condiçóes de ser saudável de pessoas e de grupos [12].

No entanto, segundo Simão et al. [12], o grau de instrução está relacionado à compreensão do indivíduo acerca do que é uma doença crônica, bem como dos fatores de risco contribuintes para sua instalação e do significado atribuído às orientações sobre alimentação, hábitos de vida e seus efeitos sobre a saúde.

Apesar de ser uma realidade, a HA não é apenas uma doença crônica. Essa falha pode ser constatada no depoimento que se segue:

É uma doença né, que não tem cura $[\ldots]$ (S11). 
Em relação à $2^{\mathrm{a}}$ questão, as ideias centrais mais frequentes foram "Responsável por doenças secundárias" e "Transtorno nas atividades da vida diária".

A HA continua sendo um grande problema de saúde em todo o mundo. Estima-se que afete aproximadamente um quarto da população brasileira. No Brasil, as doenças do aparelho circulatório constituem a principal causa de morte entre idosos, correspondendo a cerca de $40 \%$ dos óbitos dessa população. Dentre essas, as doenças cerebrovasculares e as isquêmicas do coração são as duas causas mais frequentes de óbitos. Sabe-se que a HAS é o principal fator de risco para doenças cerebrovasculares, patologia de altas taxas de morbidade, além de apresentar importante associação com doenças isquêmicas do coração e Insuficiência Cardíaca Congestiva, Insuficiência Renal e Doença Vascular Periférica [13].

Alguns participantes do estudo discorreram sobre esse assunto:

[...] minha mãe já teve AVC né, por causa da pressáo [...] (S11).

[...] No meu caso me levou a uma insuficiência renal. Em outros casos pode levar a um infarto, pode levar a um derrame [...] (S13).

Percebemos que os participantes do estudo tinham conhecimento das complicaçóes advindas da HA. As complicaçôes mais conhecidas pelos pacientes estudados sobre a doença hipertensiva, no estudo de Pierin et al. [9], foram o acidente vascular encefálico e o infarto. Apenas uma pequena porcentagem de respostas referia-se às complicaçóes renais, o que parece indicar que os sujeitos possuem um conhecimento parcial sobre essa consequência da hipertensão arterial.

De acordo com Péres, Magna \& Viana [8], grande parte dos sujeitos declarou ter medo de algumas consequências da hipertensão quando não controlada. Esse aspecto pode favorecer a adoçáo de comportamentos positivos em relaçáo aos cuidados com a doença, embora existam outros fatores intervenientes na decisão de adotar um comportamento de saúde.

Quanto à segunda ideia central frequente, evidenciam-se os seguintes discursos:

[...] quando a pressão aumenta, ela traz alguns transtornos na tua vida, ela traz muita dor de cabeça, muita dor na nuca, ela deixa a gente tontinho, parece que a gente tomou alguma bebida alcoólica, então quando ela aumenta, ela nos traz transtornos (S7).

[...] A gente fica passando mal, não consegue fazer nada direito. No começo eu sentia como se eu ficasse com água no ouvido, dor de cabeça, dor na nuca também [...] (S20).

As pessoas com doença crônica passam a experimentar diferentes sentimentos e comportamentos decorrentes de alteraçôes na capacidade física, na autoestima e na imagem corporal, nas relaçóes com outras pessoas e na realização de uma série de atividades da vida diária [14].

Firmo et al. [15] observaram, por meio de entrevista, que hipertensos pensam que a pressão alta é desencadeada especialmente por problemas familiares e pode ser facilmente reconhecida pela identificaçáo de algumas manifestaçóes específicas, associando-se explicitamente ao aparecimento de alguns problemas como tontura, mal-estar, cefaleia, aumento da pressão.

Segundo Guedes et al. [16], os sinais e sintomas apresentados pelos portadores da HA quando a mesma encontra-se em níveis elevados são cefaleia severa, sensação de mal-estar, ansiedade, agitação, tontura, dor no peito, tosse, falta de ar, alteraçóes visuais e vasoespasmos ao exame de fundo de olho.

Em relação ainda à segunda questão, um único entrevistado respondeu que a HA não afeta a saúde. Nesse sentido, elencamos a seguinte ideia central: "Tipo sanguíneo bom", para o discurso:

No meu caso não, porque tenho sangue bom [...] (S17).

Péres et al. [8] observaram que os pacientes apresentam um sistema de crenças distorcido em relação à doença hipertensiva, associado a um conhecimento parcial sobre diversos aspectos da hipertensão. Tais crenças e conhecimentos parecem gerar práticas de controle da doença também distorcidas.

Referindo-se à terceira questão, a ideia prevalente dos sujeitos que afirmaram seguir as orientaçôes recebidas foi "Manter a saúde para viver". Notamos isso nos discursos que se seguem: 
[...] quero o bem estar meu, o bem estar da saúde $[\ldots]$ procuro não exagerar em nada, nem na comida, larguei de beber, larguei de fumar e isso ajuda muito né [...] (S4).

Realizo o que falam pra mim sim, porque quero viver melhor e mais com saúde (S25).

A vontade de conhecer os aspectos da doença e do tratamento constitui fatores antecedentes à adesão ao tratamento anti-hipertensivo [17].

Portanto, além da medicação prescrita, os profissionais de saúde recomendam a adoção de práticas que possam minimizar os fatores de risco da HA. Assim sendo, o indivíduo hipertenso, para tratar ou prevenir-se das complicaçôes da HA, deve, além de medicar-se, ter atitudes para mudar antigos comportamentos ou adotar novos hábitos [18].

Ainda em relação à terceira questão, para os entrevistados que seguem as orientaçóes parcialmente, a ideia prevalente foi "Não é tão importante". O que pode ser evidenciado nos discursos:

[...] Sigo o tratamento mais ou menos [...] Não sigo certo né, por enquanto acho que não é importante seguir [...] (S6).

[...] Quando me dá irritação eu tomo, aí acho importante, quando eu fico com a pressão boa eu não tomo não porque não é importante [...] (S23).

De acordo com Araújo e Garcia [17], a experiência com um problema de saúde crônico exige a ativação de mecanismos de adaptação ao novo estilo de vida. Tal adaptação nem sempre é fácil, pois, muitas vezes, determina mudanças de hábitos prazerosos. Além disso, avaliam que enquanto os mais altos níveis de adesão relacionavam-se à medicação, os piores estavam relacionados justamente ao tratamento não-medicamentoso, incluindo dieta, atividades físicas e ingestão de álcool.

Esse comportamento denota desconhecimento sobre esse fato preocupante, tendo em vista que os objetivos do tratamento não-farmacológico são, além de reduzir as cifras tensionais, reduzir os riscos cardiovasculares.

Outra ideia evidente na terceira questáo foi “Só o remédio é suficiente”. Esta pode ser justificada no discurso a seguir:
[...] eu só tomo remédio, às vezes deixar de comer alguma coisa assim eu náo deixo, não sei por quê. Pode afetar a minha saúde, mas mesmo assim eu não deixo de comer as coisas gordurosas porque por enquanto só o remédio tá bom (S15).

É possível haver ocorrido uma tentativa dos sujeitos de negar uma parte da realidade, no caso, o tratamento náo-farmacológico, e de depositar, no médico e na medicaçáo, o poder de controlar sua pressão - como se apenas essas duas práticas fossem responsáveis pela diminuição dos níveis pressóricos. Isso poderia ser explicado pela dificuldade que muitos pacientes relatam na prática clínica em mudar um hábito ou um estilo de vida. Principalmente, considerando a existência de algumas prescrições de restriçóes altamente frustrantes, como os chamados prazeres da vida [8].

A análise dos depoimentos permitiu inferir que há uma barreira na comunicação entre equipe de saúde e portadores de HA. Isso porque percebemos um déficit de conhecimento em relaçáo às questóes básicas referentes à doença. Faz-se necessário, portanto, que os profissionais de saúde, destacando-se os enfermeiros, assistam o cliente de maneira integral adaptando as orientaçóes de enfermagem a cada realidade presenciada.

$\mathrm{Na}$ tentativa de coletar informações sobre HA emergentes de usuários cadastrados no programa HiperDia, e segundo os objetivos da presente pesquisa, foi possível estabelecer as seguintes conclusóes referentes aos portadores de HA neste estudo:

Os portadores de HA não têm conhecimento sobre a definição de sua doença, haja vista as ideias centrais mais prevalentes: "Nervosismo e preocupação", "Doença genética", "Conjunto de sinais e sintomas", "Alteração vascular", "Hábitos irregulares", "Doença ruim pelo risco de doença secundária e morte", "Doença crônica", "Uso contínuo de medicamento" e "Náo sei".

Os portadores de HA têm conhecimento dos danos que essa doença pode trazer à saúde, como se pode comprovar nas ideias centrais: "Responsável por doenças secundárias", "Uso contínuo de medicamento" e "Transtornos nas atividades da vida diária".

A única ideia que emergiu do sujeito que afirmou o motivo pelo qual a HA náo pode afetar a saúde foi "Tipo sanguíneo bom".

Os portadores de HA seguem o tratamento para o qual são orientados, como se pode perceber 
nas ideias centrais: "Manter a saúde para viver", "Em busca de cura", "Não causar transtorno" e "Para não morrer".

As ideias emergentes dos sujeitos que afirmam realizar parcialmente o tratamento para o qual são orientados foram: "Não é tão importante", "Só o remédio é suficiente" e "Tem que ter força de vontade".

Entendemos, portanto, que os profissionais da rede básica, com destaque à Estratégia Saúde da Família, são fundamentais no controle da HA, quer no diagnóstico clínico precoce e conduta terapêutica, quer nos esforços para informar, educar e conseguir que o cliente hipertenso siga o tratamento. Isso porque a mesma atua com equipe multiprofissional e tem maior vínculo com a população, levando em conta a diversidade étnica, sociocultural e outros.

Ao discutirmos que a maioria dos entrevistados não sabia a definição de HA subtende-se que há falha nas orientaçóes que a eles são fornecidas e, consequentemente, nas atividades que estão sendo desenvolvidas na Atenção Primária à Saúde.

Os portadores de HA são cadastrados pelo sistema HiperDia, porém é preciso que os profissionais entendam que apenas esse programa não é o responsável pela saúde do cliente. Além disso, notamos que o cadastramento dos hipertensos é realizado em todas as UBS. Mas o acompanhamento dessas pessoas não é feito de maneira eficaz, visto que, em grande parte das unidades ainda não há grupos de orientação para hipertensos e/ou diabéticos. As orientaçôes dadas são feitas individualmente durante a entrega mensal de medicamentos, ficando na dependência da pessoa que for à unidade ser a cadastrada ou representar algum familiar.

Portanto, a equipe da Estratégia Saúde da Família (médico, enfermeiro, técnicos e auxiliares de enfermagem e agentes comunitários de saúde), bem como outros profissionais que estejam inseridos nessa equipe (nutricionista, psicólogo e dentista) devem atuar de forma integrada e com níveis de competência bem estabelecidos, fornecendo aos seus clientes a "educação para a saúde" por meio de grupos de reuniáo. A partir desse momento, o profissional será o multiplicador da saúde, pois as pessoas que receberão as informaçóes corretas irão transmiti-las a outros indivíduos. Além do acompanhamento dos pacientes hipertensos, os grupos devem ser facilitadores para que ocorra o conhecimento da doença e a adesão ao tratamento, lembrando que todos os indivíduos devem receber atendimento personalizado. O profissional da saúde não pode se acomodar diante da primeira dificuldade encontrada em relaçáo ao paciente hipertenso, visto que é preciso tempo para que o mesmo se adapte a nova situação de vida.

Porém, para fazer com que essa educação realmente torne-se eficaz, é preciso que os profissionais desenvolvam seu trabalho com motivação e criatividade, porque é somente dessa maneira que os clientes sentir-se-ão motivados em querer cuidar de si mesmos.

\section{Conclusão}

A análise e a discussão realizadas nesta pesquisa demonstram que se faz necessário, por parte dos enfermeiros:

Capacitar à equipe de enfermagem e os agentes comunitários de saúde para que os mesmos transmitam informaçóes pertinentes sobre HA;

Organizar grupos de educação para a saúde, bem como fornecer informaçóes individuais sempre que surgirem oportunidades;

Realizar consultas de enfermagem, desenvolvendo intervençóes frente à realidade de cada indivíduo hipertenso.

\section{Referências}

1. Ministério da Saúde. HiperDia - Sistema de Cadastramento e Acompanhamento de Hipertensos e Diabéticos. Brasília: Ministério da Saúde; 2002.

2. Ministério da Saúde. Sistema de Vigilância em Saúde. Sistema de Informaçóes sobre Mortalidade [online]. [citado 2008 Nov 12]. Disponível em URL: http:// tabnet.datasus.gov.br

3. Sociedade Brasileira de Hipertensão. V Diretrizes Brasileiras de Hipertensão Arterial. Hipertensão 2006;9:126-57.

4. Machado SC, Stipp MAC, Leite JL. Clientes com hipertensão arterial: perspectiva da gerência do cuidado de enfermagem. Esc Anna Nery Rev Enferm 2005;9:64-71.

5. Moscovi S. Representação social da psicanálise. Rio de Janeiro: Zahar; 1978.

6. Lefrève F, Lefrève AM. Discurso do sujeito coletivo. São Paulo: Eduso; 2004

7. Cavalini LT, Chor D. Inquérito sobre hipertensão arterial e déficit cognitivo em idosos de um serviço de geriatria. Rev Bras Epidemiol 2003;6:7-17.

8. Péres DS, Magna JM, Viana LA. Portador de hipertensão arterial: atitudes, crenças, percepçóes, pensamentos e práticas. Rev Saúde Pública 2003;37:635-42. 
9. Pierin AMG, Junior Mion D, Fukushima JT, Pinto AR, Kaminaga MM. O perfil de um grupo de pessoas hipertensas de acordo com conhecimento e gravidade da doença. Rev Esc Enferm USP 2001;35:11-8.

10. Miranda RD, Perrotti TC, Bellinazzi VR, Nobrega TM, Cendoroglo MS, Neto JT. Hipertensão arterial no idoso: peculiaridades na fisiopatologia, no diagnóstico e no tratamento. Rev Bras Hipertens 2002;9:293-300.

11. Silva LF, Guedes MVC, Moreira RP, Souza ACC. Doença crônica: o enfrentamento pela família. Acta Paul Enferm 2002;15:40-7.

12. Simão M, Nogueira MS, Hayashida M, Cesarino EJ. Doenças cardiovasculares: perfil de trabalhadores do sexo masculino de uma destilaria do interior paulista, 2002. Rev Eletrônica Enferm 2002;4(2):27-35.

13. Ministério da Saúde. Secretaria de políticas de saúde. Plano de reorganização da atenção à hipertensão arterial e ao diabetes mellitus. Manual de hipertensão arterial e diabetes mellitus. Brasília: Ministério da Saúde; 2002. p. 1-102.
14. Martins LM, França APD, Kimura M. Qualidade de vida de pessoas com doença crônica. Revista Latinoam Enferm 1996;4(3):5-18.

15. Firmo JOA, Lima-Costa MF, Uchôa E. Projeto Bambuí: maneira de pensar e agir de idosos hipertensos. Cad Saúde Pública 2001;20:1029-40.

16. Guedes NG, Costa FBC, Moreira RP, Moreira TF, Chaves ES, Araújo TL. Crises hipertensivas em portadores de hipertensão arterial em tratamento ambulatorial. Rev Esc Enferm USP 2005;39:181-8.

17. Araújo GBS, Garcia T R. Adesão ao Tratamento Anti-hipertensivo: uma Análise Conceitual. Rev Eletrônica de Enferm 2006;8:259-72.

18. Lima MT, Bucher JSMF, Lima JWO. A hipertensão arterial sob o olhar de uma população carente: estudo exploratório a partir dos conhecimentos, atitudes e práticas. Cad Saúde Pública 2004;20:1079-87. 